\title{
High prevalence of sexually transmitted diseases in a rural area in Mozambique
}

Bea Vuylsteke, Rui Bastos, Jorge Barreto, Tania Crucitti, Elena Folgosa, José Mondlane, Tine Dusauchoit, Peter Piot, Marie Laga

\begin{abstract}
Objective-To assess the extent of the sexually transmitted diseases (STDs) problem in a rural area of Mozambique. Methods-A cross sectional study among pregnant women and patients presenting with genital complaints. Laboratory confirmation was done for gonorrhoea, chlamydial infection, active syphilis, trichomoniasis and HIV infection.

Setting-A primary health care setting in Vilanculos, Inhambane province, Mozambique.

Results-Evidence of one or more of the above STDs was found in 51\% of 201 pregnant women, $56 \%$ of 85 women and $62 \%$ of 77 men with genital complaints. Neisseria gonorrhoea or Chlamydia trachomatis were found in $16 \%$ of pregnant women, $23 \%$ of female patients and $28 \%$ of male patients; genital ulcer disease was present in $6 \%, 28 \%$ and $36 \%$, of respectively pregnant women, female and male patients. The prevalence of active syphilis was about the same in the three groups of study subjects, that is $15 \%$. HIV infection was found in $4 \%$ of the male patients; no HIV infection could be detected in the female groups.

Conclusion-STDs were a major health problem in this rural area in Mozambique. Though HIV infection was still low, the high prevalence of STDs indicates that the potential is there for an explosive spread of the HIVIAIDS epidemic.
\end{abstract}

(Genitourin Med 1993;69:427-430)

\section{Introduction}

Sexually transmitted diseases (STDs) are more frequent in many developing country populations than in the developed world. In recent years, evidence has been accumulating that the classical STDs facilitate the transmission of HIV infection. ${ }^{1}$ Control of gonorrhoea, chlamydial infection and genital ulcer disease (GUD) would not only prevent complications such as pelvic inflammatory disease and tubal occlusion in women and congenital syphilis in infants, but might also slow down the spread of HIV infection. ${ }^{2}$

Whereas numerous studies have been conducted on the prevalence of STDs in urban populations of developing countries, ${ }^{3}$ there is a paucity of data on the frequency of STDs in rural areas. In 1991, the non governmental organisation Médecins Sans Frontières reported that about $10 \%$ of attendances at primary health care (PHC) facilities in the north of Inhambane province were for STD-related complaints. In order to assess the extent of the STD problem in this area, a PHC facility based study was performed among pregnant women and patients with genital complaints.

The objectives of the study were: (1) to estimate the frequency of STD-related complaints among patients attending the PHC facility, (2) to assess the prevalence of STD in women attending the antenatal clinic (ANC), (3) to compare the proportions of different STDs in the three study populations, pregnant women, male and female patients, (4) to assess the percentage of penicillase producing $N$ gonorrhoeae (PPNG) strains.

\section{Materials and methods}

Setting and study population

The study was conducted in Vilanculos, a small littoral town with a population of 18000 , in the north of Inhambane province. Until the peace agreement of October 1992, communication with larger towns and the capital city Maputo was exclusively through military convoys. Fishing is the most important economic activity. The PHC facility is situated in the compound of the district hospital which has laboratory facilities. Between mid December 1991 and February 1992, every other pregnant woman visiting the ANC and all patients over the age of 15 years with genital complaints were enrolled in the study. The following symptoms were considered as being STD-related: urethral discharge, dysuria, itching of the glans in men, vaginal discharge, dyspareunia, vulvo-vaginal irritation, pelvic pain in women, and genital ulcers, inguinal bubo, anogenital warts in both sexes.

The pregnant women were interviewed and examined by a Mother and Child Health $(\mathrm{MCH})$ nurse, the patients by a physician (BV). The interview included questions about age, marital status, sexual behaviour, and STD symptoms. Clinical examination in women included speculum examination and bimanual palpation. A vaginal smear and an endocervical swab were taken from the women, a urethral swab was taken from the men. In addition, a blood sample was taken. Informed verbal consent was obtained from every participant in the study. 


\section{Laboratory procedures}

Infection by Trichomonas vaginalis was diagnosed by direct microscopic examination of the vaginal smear. Urethral and cervical specimens were cultured for $N$ gonorrhoeae by inoculation on modified Thayer Martin medium followed by incubation in a candle extinction jar at $35^{\circ}$ for $24-48$ hours. Isolates were identified on typical colonial morphology, oxidase reaction and Gram stain. PPNG strains were identified in vitro by Beta Lactamase Detection papers (Oxoid, Basingstoke, England). Other urethral and cervical specimens were cultured for $C$ trachomatis on Mc Coy cells in Antwerp.

All sera were screened for syphilis by rapid plasma reagin (RPR, Becton Dickinson, Cockeysville, Maryland US), and reactive sera were subsequently tested by Treponema Pallidum Hemagglutination test (TPHA, Fujirrbio, Tokyo, Japan). The combination of a reactive RPR and reactive TPHA test was considered as an indication of present or recently past syphilis.

HIV antibody was detected in a rapid test (Testpack HIV1/HIV2, Abott GmbH, Delkenheim, Germany); all sera were tested with Elisa (Elavia Mixt, Diagnostics Pasteur, Marnes-la Coquette, France) and positive results were confirmed by Western Blot (HIV-1 Western Blot IgG assay, Diagnostic Biotechnology Ltd, Singapore, Singapore and Lav-Blot II, Diagnostics, Pasteur, Marnes-la Coquette, France).

Anti-Haemophilus ducreyi serum immunoglobulin IgG were detected by an enzyme immunoassay (EIA), on a crude cocktail antigen of lysates from eight different immunotypes of $H$ ducreyi. ${ }^{3}$ Sera with an optical density [O.D.]/[cut off O.D.] ratio of $>1$ were considered as positive.

Examination of wet mount, culture for gonorrhea, RPR and rapid test for HIV infection were done in Vilanculos. TPHA, Elisa and Western Blot were done in Maputo, in respectively the reference laboratory for microbiology and the HIV reference laboratory. Culture for $C$ trachomatis and testing for anti- $H$ ducreyi antibodies were done at the Institute of Tropical Medicine in Antwerp. Samples were transported to Maputo and to Antwerp in liquid nitrogen.

\section{Statistical methods}

Data were analysed in EPI-INFO. Bivariate analysis was performed using Yates' corrected chi-square.

\section{Results}

\section{Characteristics of the study populations}

Pregnant women were younger and were more likely to have a stable relationship than patients. Out of the 81 female patients, 12 $(15 \%)$ were widowed or separated, whereas this percentage was only $2 \%$ and $3 \%$ respectively in the pregnant women and the men. Reliable contraceptive methods were taken only by 5 of the 81 female patients. Fifty three percent of the male patients admitted to have had more than five sexual partners in the last 6 months, versus $4 \%$ of the female patients. Although $89 \%$ of the men knew what a condom is, only $26 \%$ had ever used one. Nearly half of female patients had ever seen a condom, and only $6 \%$ had ever used one.

Out of the 1439 patients over the age of 15 years who consulted the PHC facility during the study period, 155 (11\%) presented with STD related symptoms. Five of them persuaded their partner to come for treatment.

On questioning, only $6 \%$ of pregnant women reported a symptom, mostly vaginal discharge. Among the female patients, vaginal discharge was also the most common complaint $(63 \%)$, followed by lower abdominal pain $(51 \%)$ and genital ulceration $(21 \%)$. In men, $50 \%$ complained of urethral discharge and $35 \%$ complained of genital ulceration. Twelve men presented themselves with other symptoms like dysuria without discharge and itching of the glans.

\section{STD prevelance rates}

Evidence of one or more STDs was found in $51 \%$ of pregnant women, $59 \%$ of female patients and $65 \%$ of male patients. The prevalences of the different STDs in the three study populations are summarised in the Table. Of the pregnant women, $7 \%$ had gonorrhoea, $8 \%$ chlamydial infection, $15 \%$ active syphilis and $23 \%$ trichomoniasis; genital ulceration was found in $6 \%$ of them. The prevalences of all STDs, except GUD were similar in the female patients and the pregnant women (GUD $28 \%$ vs. $6 \%, \mathrm{p}<0.001$ ). The male patients had less chlamydial infections than the female patients ( $7 \%$ vs. $12 \%)$. Syphilis was equally prevalent in both sexes $(15 \%)$. Other STDs were more frequent in the men than in the women: gonorrhoea 22 vs. $12 \%$, GUD 38 vs $28 \%$, for men and women respectively. Chlamydia infection and syphilis were associated with lack of circumcision (respectively OR $19, p=0.001$ and OR $4, p=0.04)$. This association was not found with GUD or with other STDs.

Table 1 STD prevalences in the three study populations

\begin{tabular}{|c|c|c|c|}
\hline \multirow[b]{2}{*}{ Prevalence } & \multirow{2}{*}{$\begin{array}{l}\text { Pregnant } \\
\text { women } \\
n=201 \%\end{array}$} & \multicolumn{2}{|c|}{ Selected patients } \\
\hline & & $\begin{array}{l}\text { women } \\
n=81 \%\end{array}$ & $\begin{array}{l}m e n \\
n=74 \%\end{array}$ \\
\hline$N$ gonorrhoeae & $\begin{array}{l}7 \cdot 0 \\
(3 \cdot 5-10 \cdot 5)\end{array}$ & $\begin{array}{l}12 \cdot 3 \\
(5 \cdot 2-19 \cdot 4)\end{array}$ & $\begin{array}{l}21 \cdot 6 \\
(12 \cdot 2-31)\end{array}$ \\
\hline$C$ trachomatis $^{\circ}$ & $\begin{array}{l}7 \cdot 9 \\
(3 \cdot 5-12 \cdot 3)\end{array}$ & $\begin{array}{l}12 \cdot 0 \\
(4 \cdot 7-19 \cdot 3)\end{array}$ & $\begin{array}{l}7 \cdot 1 \\
(1 \cdot 1-13 \cdot 1)\end{array}$ \\
\hline G.U.D.* & $\begin{array}{l}6 \cdot 0 \\
(2 \cdot 7-9 \cdot 3)\end{array}$ & $\begin{array}{l}28 \cdot 4 \\
(18 \cdot 6-38 \cdot 2)\end{array}$ & $\begin{array}{l}37 \cdot 8 \\
(26 \cdot 8-48 \cdot 8)\end{array}$ \\
\hline Syphilis & $\begin{array}{l}14 \cdot 6 \\
(9 \cdot 7-19 \cdot 5)\end{array}$ & $\begin{array}{l}14 \cdot 9 \\
(7 \cdot 1-22 \cdot 5)\end{array}$ & $\begin{array}{l}14.9 \\
(6 \cdot 8-23)\end{array}$ \\
\hline Trichomonas vaginalis & $\begin{array}{l}22 \cdot 8 \\
(16 \cdot 9-28 \cdot 7)\end{array}$ & $\begin{array}{l}24 \cdot 4 \\
(14 \cdot 9-33 \cdot 9)\end{array}$ & \\
\hline None of the above & $\begin{array}{l}49 \cdot 4 \\
(42 \cdot 5-56 \cdot 3)\end{array}$ & $\begin{array}{l}40 \cdot 7 \\
(30-51 \cdot 4)\end{array}$ & $\begin{array}{l}35 \cdot 1 \\
(24 \cdot 2-46)\end{array}$ \\
\hline Antibodies $H$ ducreyi & $\begin{array}{l}20 \cdot 4 \\
(14 \cdot 8-26)\end{array}$ & $\begin{array}{l}33 \cdot 3 \\
(23-43 \cdot 6)\end{array}$ & $\begin{array}{l}35 \cdot 1 \\
(24 \cdot 2-46)\end{array}$ \\
\hline
\end{tabular}

?95\% confidence intervals.

${ }^{\circ}$ :results of $C$ trachomatis culture on total of 141 pregnant women, 78 of the female and 73 of the male patients. *G.U.D. = Genital Ulcer Disease tRPR + and TPHA + 
Twenty per cent of the female and $11 \%$ of the male patients had concomitant infections. The most common dual infection was gonorrhoea with syphilis: one third of all cases of gonorrhoea also had active syphilis. HIV infection was confirmed in three men, including two with HIV1 and one with HIV2 infection.

Of the 42 strains of $N$ gonorrhoeae tested, $27(64 \cdot 3 \%)$ were beta-lactamase positive.

\section{Discussion}

These findings suggest a very high prevalence of STDs in the general population of Vilanculos, a district which may be representative for rural areas along the 2500 kilometre long coast of Mozambique. The prevalence of active syphilis in pregnant women which was found by Liljestrand in six of the country's 10 provinces ranged from $1.6 \%$ (Muiane, community based survey) to $\mathbf{9 . 8 \%}$ (antenatal care unit of a suburb of Maputo). ${ }^{4}$ A possible explanation for the $15 \%$ which we found could be the collapse of health services during the civil war. High prevalences of syphilis in pregnant women have been reported from other Southern and Eastern Africa countries, such as Swaziland (13\% in 1988), ${ }^{5}$ South Africa $(12 \% \text { in } 1989)^{6}$ and Tanzania $(11 \%$ in 1990) (Riedner, personal communication); this is in contrast with the low prevalences that have been found in Central and Western African countries like Zaire (1\% in 1990), ${ }^{7}$ and Côte d'Ivoire ( $1 \%$ in 1992)..$^{8}$ The prevalence of gonorrhoea (7\%) was also higher than that found in most studies from other African countries. ${ }^{7-9}$ On the other hand, the frequency of chlamydia infection was similar to what was found elsewhere ${ }^{79}$. The near total absence of symptoms in these pregnant women precludes a screening program for STDs based on an approach by symptoms alone.

The ratio of gonorrhoea to chlamydia infection was higher in male patients than in female patients ( 3 vs. 1 ). A possible explanation for this could be auto-medication. Men with urethritis would first buy antibiotics like tetracycline on the market, which would clear a chlamydia infection, but not gonorrhoea. The high prevalence of $H$ ducreyi antibodies in this population suggests that chancroid was responsible for a large proportion of the GUD's seen. This assumption is consistent with findings in Swaziland, where the diagnosis of chancroid was made in $42 \%$ of genital ulcers. ${ }^{10}$ In South Africa, 22\% of the GUD in men $^{11}$ and $14 \%$ of the GUD in women ${ }^{12}$ were found to be chancroid. Other possible etiologies of GUD in this region include: genital herpes, donovanosis, and lymphogranuloma venereum. ${ }^{11} 12$

The high frequency of STD-related complaints among patients attending PHC services $(11 \%)$ underscores the importance of proper STD case detection and management at this level of health care.

Diagnosis of STDs in the absence of sophisticated laboratory facilities remains difficult, especially in women, because of the low sensitivity and specificity of symptoms. As mixed infections are very common, syphilis screening for every STD patient should be envisaged.

Health care workers in Mozambique routinely advise STD patients to notify and refer their partner for treatment. In our study, five patients brought their partners for treatment. If more emphasis were placed on partner referral, this number probably could be increased.

The high proportion of PPNG strains (64\%) we found confirms an earlier report from Maputo, where $43 \%$ of gonococcal strains were found to be PPNG (1990, E Folgosa, personal communication).

The low prevalence of HIV infection contrasts with the prevalences of the other STDs. Only three male patients (3.9\%) and none of the females were confirmed HIV positive. Because of the war, Mozambique has been relatively isolated from neighbouring countries such as Malawi, Tanzania and Zambia, which have a high prevalence of HIV infection. ${ }^{13}$ However, high prevalences of STDs in the range that we found suggest that in Mozambique circumstances are favourable for an explosive HIV epidemic, especially now that the civil war has come to an end and roads have been opened again. In Maputo, HIV seroprevalence in STD patients rose from $0.4 \%$ in 1990 to $3.1 \%$ in 1991 , and numbers of reported AIDS cases nationwide increased from 1 in 1986 to 98 in 1990 and 178 in 1991 (Seventh report of the National Aids Program, Ministry of Health, Mozambique, 1992).

Health education and condom promotion are still in their early stages in rural areas. This was evidenced by the low percentage of women $(43 \%)$ who had ever seen a condom in their life. Male patients were more familiar with condoms, but the utilization rate was low. Apart from the problem of acceptability, condoms were not readily available and accessible in this village.

In conclusion, because of the very high STD rates in Mozambique and the large potential for the spread of HIV infection, STD control and management assume top priority. Case management at primary health care level provides a unique opportunity for educating people with high risk behaviour. The case detection rate for syphilis can be substantially increased by routine screening of pregnant women and patients presenting with an STD.

We thank all collaborators in Vilanculos: Enrique Munoz (MSF), Maria Laice, Gilda Florencia Guerra and Lourenço Langa (Primary Health Care), Ruben Sebastiao Bombe (laboratory) and the MSF logistic team. Our special thanks go to Eddy Van Dyck and Erwin Roggen of the laboratory of to Eddy Van Dyck and Erwin Roggen of the

This study was funded in part by Médecins sans Frontieres, Belgium.

1 Wasserheit J. Epidemiological synergy, Interrelationship between Human Immunodeficiency Virus Infection and Other Sexually Transmitted Diseases. Sex Transm dis 1992;19:61-77. 
2 Laga M, Manoka A, Kivuvu M, Malele B, et al. Nonulcerative Sexually Transmitted Diseases as risk factors for HIV-1 transmission in women. Results from a cohort study. AIDS 1993;7:95-102.

3 Roggen E, De Breucker S, Van Dijck E, Piot P. Antigenic diversity in Haemophilus ducreyn as shown by Western Blot (Immunoblot) analysis. Infect Immun 1992;60: 590-5.

4 Lijestrand J, Bergstrom S, Nieuwenhuis F, Hederstedt B. Syphilis in pregnant women in Mozambique. Genitourin Med 1985;61:355-8.

5 Guinness LF, Sibandze S, McGrath E, Cornelis et al. Influence of antenatal screening on perinatal mortality caused by syphilis in Swaziland. Genitorin Med 1988;64: 294-7.

6 O'Farrell N, Hoosen AA, Kharsany ABM, Van den Ende J. Sexually transmitted pathogens in pregnant women in a rural South African community. Genitourin Med 1989;65:276-80.

7 Luyeye M, Gerniers M, Lebughe N, et al. Prevalence et facteurs de risque pour les MST chez les femmes enceintes dans les soins de santé primaires á Kinshasa.
Fifth International Conference on AIDS in Afrika 1990, Kinshasa; Abstract T.P.C.8

8 Diallo MO, Traore V, Maran M, et al. Sexually transmitted diseases and HV1/2 infections among pregnan women attending an antenatal clinic in Abidjan, Côte d'Ivoire. VIIth International Conference on AIDS in Africa 1992, Cameroun; Abstract TP041.

9 De Schampheleire I, Van De Velden L, Van Dijck E, et al. Maladies sexuellement transmissibles dans la population féminine á Pikine, Sénégal. Ann Soc belge Méd trop 1990;70:227-35.

10 Meheus A, Van Dyck E, Ursi JP, et al. Etiology of genital ulcerations in Swaziland. Sex Transm Dis 1983;10:33-5.

11 O'Farrell N, Hoosen AA, Coetzee KD, Van den Ende J. Genital ulcer disease in men in Durban, South Africa. Genitourin Med 1991;67:327-30.

12 O'Farrell N, Hoosen AA, Coetzee KD, Van den Ende J. Genital ulcer disease in women in Durban, South Africa. Genitourin Med 1991;67:322-6.

13 Nkowane BM. Prevalence and incidence of HIV infection in Africa: a review of data published in 1990. AIDS 1991;5 (suppl 1):S7-S15. 